\title{
Experimental Characterization of Back-Scattering Interference Limits for Fronthaul employing Bitrate Variable Transceivers based on OFDM and Direct Detection
}

\author{
Josep M. Fabrega, Michela Svaluto Moreolo, Laia Nadal \\ Centre Tecnologic de Telecomunicacions de Catalunya (CTTC/CERCA), Av. Carl F. Gauss, 7; Castelldefels, Spain. \\ jmfabrega@cttc.es
}

Abstract: We experimentally assess the impact of back-scattering when featuring DDOFDM bidirectional transmission over a single fiber. Results show that different functional split options can be supported for signal/scattering ratios $>16 \mathrm{~dB}$ and received optical power around $-24 \mathrm{dBm}$.

OCIS codes: (060.4510) Optical communications; (060.4250) Networks.

\section{Introduction}

In $5 \mathrm{G}$, a high capacity demand is required together with a dynamic network management. In fact, a large number of antenna elements over small cells are envisioned for increasing the data rate per end user [1]. Therefore, the 5G network paradigm is expected to have a segment close to the end user supported by wireless communications, while optical fiber links serve the corresponding radio access network (RAN) which demands very high capacity [1].

A popular solution is the so-called centralized RAN (C-RAN) featuring digital fronthaul [2]. There, a pool of broadband units (BBUs) implementing all radio signal generation and further digitization is placed in a central office (CO), while simple remote radio units (RRUs) are scattered at the other end of the RAN (see Fig.1a). This solution entails a high capacity and strict latency requirement [1]. Typical capacities are higher than 10Gb/s and maximum transmission latencies are less than hundreds of $\mu$ s, limiting the transmission distance to less than $25 \mathrm{~km}$ of standard single mode fiber (SSMF) [1]. In order to relax these strict requirements, 3GPP is proposing to perform a function split of the baseband processing with eight different options [3]. In fact, the BBU functions are split into two logical entities: central unit (CU, e.g. located at the CO) and distributed unit (DU, e.g. located at the RRU). The split options under consideration in 3GPP can be categorized as high layer (options 2 and 3), medium layer (options 5 and 6), and low layer (options 7 and 8), all of them with different latency and capacity requirements [3].

Since C-RAN deployment should be covered at an affordable cost, a suitable solution can be the reuse of already deployed fiber infrastructure in the residential access, as suggested in [1]. In this sense, NGPON2 has included the point to point (PtP) wavelength division multiplexed (WDM) scheme in order to allow a WDM overlay of additional services (including C-RAN) together with fixed access [4].

Regarding optical transmission technologies, bandwidth/bitrate variable transceivers (BVTs) can be employed for such a C-RAN deployment, providing the required flexible connectivity and scalable capacity [5]. This also enables a transparent connection to the metro/aggregation segment as suggested in [5]. A very dense WDM scheme is proposed with BVTs based on orthogonal frequency division multiplexing (OFDM) and direct detection (DD). Therefore, the capacity of the transceivers can be configured (e.g. by an SDN controller) according to the network condition, including transmission impairments and the suitable functional split option. Interestingly, OFDM features adaptive modulation of its subcarriers, so the depth of modulation can be adapted to the channel spectral response. Furthermore, the BVTs are a fundamental building block of the so-called sliceable BVT (S-BVT), where different WDM traffic flows are generated/detected in order to either serve the same site or concurrently serve different locations [6]. Since capacity is expected to be further increasing, and OFDM-based (S-)BVTs can adapt the transmission to the channel response, a highly dense WDM deployment can be approached for C-RAN when envisioning a bidirectional transmission over a single fiber using the same wavelength for upstream and downstream.

This paper presents an experimental assessment of single fiber bidirectional transmission of DD-OFDM BVTs over $25 \mathrm{~km}$ of SSMF employing the same wavelength for upstream and downstream. Taking advantage of the OFDM adaptive modulation, we explore its capacity limits in terms of sensitivity and optical signal to interferent back-scattering ratio (SIR) level due to the counter-propagating signal. Results show that low layer functional split options can be supported for SIR values above $16 \mathrm{~dB}$ and covering a power budget of $20 \mathrm{~dB}$.

2. Experimental setup

The experimental setup is shown in Fig.1b. The digital signal processing (DSP) and electrical up/down-conversion at the transmitter of each transmitted signal is performed off-line, following the steps are detailed in [6] (insets of 


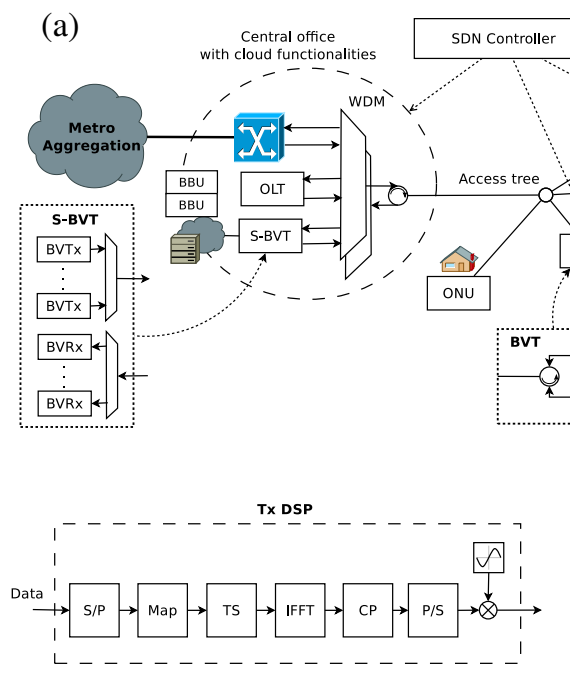

Fig. 1. (a) General scheme. (b) Experimental setup.

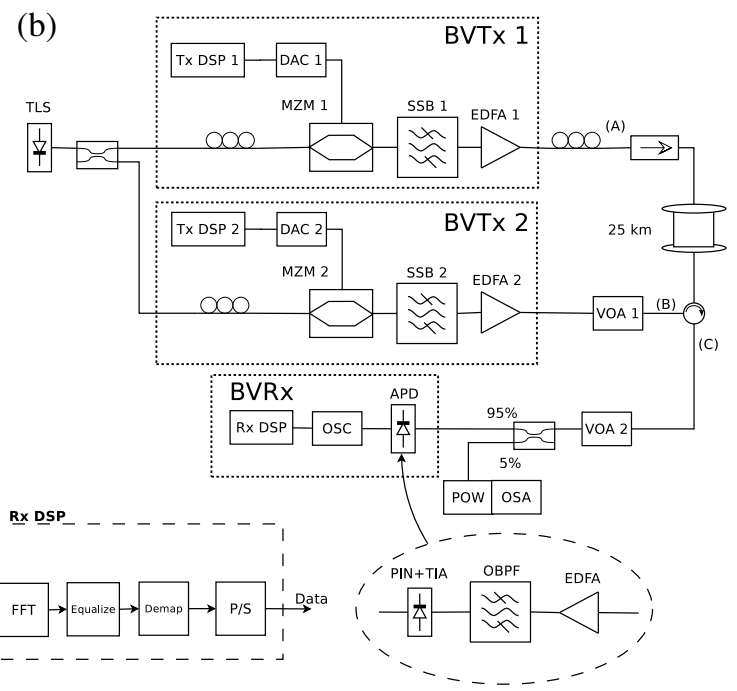
Fig.1). Tx DSP 1 corresponds to the useful signal and features adaptive bit/power loading using the Levin-Campello
rate adaptive algorithm [6] in order to test the maximum capacity that the system can offer. Tx DSP 2 is running at a fixed data rate $(40 \mathrm{~Gb} / \mathrm{s})$ for generating an interferent signal. The OFDM signal after each $64 \mathrm{GSa} / \mathrm{s}$ digital to analogue converter (DAC) is fixed to be running at $20 \mathrm{Gbaud}$ and centered to $10 \mathrm{GHz}$. This signal drives the corresponding Mach-Zehnder modulator (MZM) biased at the quadrature point. Both MZMs are excited by the same tunable laser source (TLS) working at $1549.99 \mathrm{~nm}$. A polarization controller is present at the input of each MZM in order to attain an optimum modulation. The resulting optical signals pass through the corresponding optical filter $(25 \mathrm{GHz}$ bandwidth and centered at $1550.12 \mathrm{~nm}$ ) for obtaining an optical single sideband (SSB) signal. After each SSB filter, an erbium doped fiber amplifier (EDFA) at each arm compensates the losses after SSB filtering. A sample spectrum of the transmitted signal is shown in Fig.2a. The power injected to the fiber is $-4 \mathrm{dBm}$ at point (A) of Fig.1. In (B) the power is varied between $-5 \mathrm{dBm}$ and $+10 \mathrm{dBm}$ by means of a variable optical attenuator (VOA), being slightly below the transmission power limits specified for PtP WDM in NGPON2 [4]. The optical signal to noise ratio (OSNR) measured within 0.1nm is $31.2 \mathrm{~dB}$ for the worst case (after EDFA 2).

The generated signals are injected to a $25 \mathrm{~km}$ SSMF in opposite directions. Therefore, an interferent signal is present at point (C), mainly contributed by Rayleigh and Brillouin back-scattering of the signal present in (B). The SIR can be varied by tuning the attenuation of VOA 1. Fig.2d shows sample spectra acquired after (C) for different SIR levels when no signal is present at point (A). There we can see a spectrum similar to the transmitted one (shown in Fig.2a) due to Rayleigh back-scattering, but with two peaks spaced about $11 \mathrm{GHz}$ with respect to the main peak due to Brillouin back-scattering.

In order to isolate the interferent signal, an optical isolator is present at point (A), while an optical circulator is placed between the optical fiber and points (B) and (C). After the circulator, a VOA and a 95:5 power splitter are present in order to vary and measure the optical power at the input of the receiver. Afterwards a high bandwidth avalanche photodiode is emulated by the combination of gain-stabilized EDFA, optical band pass filter (OBPF) and PIN diode. This combination is calibrated to obtain a $-28 \mathrm{dBm}$ sensitivity for $10^{-3}$ bit error ratio (BER) for on-off keying transmission at $10.7 \mathrm{~Gb} / \mathrm{s}$, as in [5]. The photodetected current is digitized by a real-time oscilloscope (OSC) running at $100 \mathrm{GSa} / \mathrm{s}$. Finally, the baseband OFDM signal is recovered after downconversion and off-line demodulated according to [6]. BER is calculated by error counting.

\section{Results}

For all the results reported, the BER obtained is within the range between $10^{-3}$ and $4.62 \cdot 10^{-3}$, corresponding to the threshold values for a standard hard decision forward error correction (FEC) featuring 7\% overhead [7].

Regarding the functional split options analyzed, a wireless channel is assumed with 100MHz bandwidth, 256QAM modulation, and $8 \times 8$ MIMO. Details can be found in Table A-1 of [3]. There, the minimum latency requirement is $250 \mu$ s (split options 7 and 8), while the nominal propagation delay of the SSMF spool used is of $122.4 \mu \mathrm{s}$.

First, the back-to-back (B2B) and transmission over $25 \mathrm{~km}$ are characterized without any back-scattering. So, no signal is present at point (B) of Fig.1. Results are depicted in Fig.2b. Fig.2b shows that, at $-15 \mathrm{dBm}$, the capacity attained is $54.9 \mathrm{~Gb} / \mathrm{s}$ for the B2B and $45.1 \mathrm{~Gb} / \mathrm{s}$ after $25 \mathrm{~km}$. So, some cases of split options $7 \mathrm{~b}$ and $7 \mathrm{c}$ (requiring up to $53.8 \mathrm{~Gb} / \mathrm{s}$ ) could be supported only in B2B. This capacity decreases with the received optical power. At $-20 \mathrm{dBm}$, 

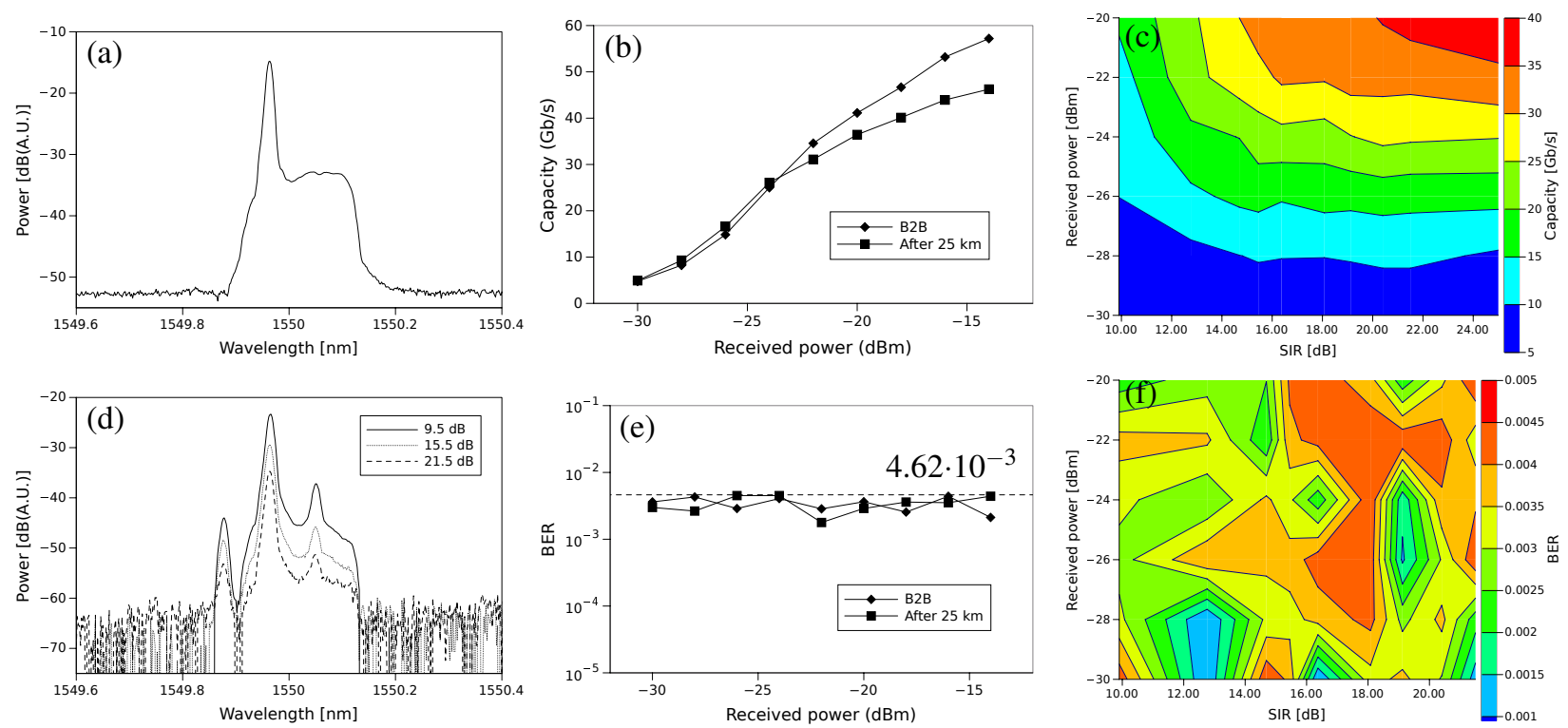

Fig. 2. (a) Sample spectrum of transmitted signal. (b) Capacity vs received power with no backscattering and (e) the corresponding BER. (c) Capacity as function of the SIR and received power after $25 \mathrm{~km}$ SSMF, and (f) the corresponding BER. (d) Back-scattered spectra at different SIR.

the maximum capacity attained is $41.1 \mathrm{~Gb} / \mathrm{s}$ for the $\mathrm{B} 2 \mathrm{~B}$ and $36.4 \mathrm{~Gb} / \mathrm{s}$ after propagating through $25 \mathrm{~km}$ of optical fiber. Therefore, the maximum functional split supported in this case is option $7 \mathrm{a}$, which needs a maximum of $22.2 \mathrm{~Gb} / \mathrm{s}$ [3]. Such split option can be supported down to $-24 \mathrm{dBm}$ of received power, where B2B and transmission after $25 \mathrm{~km}$ feature a capacity around $25 \mathrm{~Gb} / \mathrm{s}$. The lower power measured is $-30 \mathrm{dBm}$, where both configurations feature a capacity slightly higher than $5 \mathrm{~Gb} / \mathrm{s}$. In this case, the maximum functional split supported is option 5 , requiring up to $4 \mathrm{~Gb} / \mathrm{s}$.

Next, the signal coming from MZM 2 is activated and the interference effect from back-scattering is characterized. Results are shown in Fig.2c. In Fig.2c we can see that SIR is limiting the performance also in terms of capacity. In fact, option 7a of functional split can be supported for SIR values $>16 \mathrm{~dB}$ and power values around $-24 \mathrm{dBm}$, similar to the results reported in B2B and $25 \mathrm{~km}$ with no back-scattering. For SIR values below $16 \mathrm{~dB}$, the capacity decreases and a worst case for supporting split option $7 \mathrm{a}$ is found at $14 \mathrm{~dB}$ SIR and $-20 \mathrm{dBm}$ received power. At $-30 \mathrm{dBm}$ of received power, capacity is slightly higher than $5 \mathrm{~Gb} / \mathrm{s}$ for all SIR values, supporting the functional split option 5 .

The split option requiring higher capacity is option 8 , asking for $157.3 \mathrm{~Gb} / \mathrm{s}$. So, a sliceable BVT configuration with at least 7 flows (wavelengths) is needed to meet this requirement at SIR values $>16 \mathrm{~dB}$ and $-24 \mathrm{dBm}$ received power.

\section{Conclusions}

The impact of back-scattering when featuring DD-OFDM bidirectional transmission over a single fiber is assessed for different functional split options. Low layer options (up to 7a) can be easily served with a single BVT. Thus, BVTs based on DD-OFDM is a promising solution for serving different sites when featuring a flexible functional split.

Work supported by MICINN (TEC2015-69256-R, RTI2018-099178-B-IO0) and EU H2020 BLUESPACE (G.A. 762055) projects

\section{References}

1. B. Skubic et al. "Optical transport solutions for $5 \mathrm{G}$ fixed wireless access," Journal of Optical Communications and Networking, vol. 9, no. 9, p. D10 (2017)

2. T. Pfeiffer, "Next Generation Mobile Fronthaul and Midhaul Architectures," Journal of Optical Communications and Networking, vol. 7, no. 11, p. B38 (2015)

3. 3GPP TR 38.801 V14.0.0 "Technical Specification Group Radio Access Network; Study on new radio access technology: Radio access architecture and interfaces" (2017)

4. D. Nesset, "NG-PON2 Technology and Standards," J. Lightwave Technol., Vol. 33, no. 5, p. 1136 (2015).

5. J. M. Fabrega et al., "Experimental Validation of a Converged Metro Architecture for Transparent Mobile Front/Back-Haul Traffic Delivery Using SDN-Enabled Sliceable Bitrate Variable Transceivers," J. Lightwave Technol., Vol. 36, no. 7, p. 1429 (2018).

6. M. Svaluto Moreolo et al., "SDN-enabled Sliceable BVT Based on Multicarrier Technology for Multi-Flow Rate/Distance and Grid Adaptation," J. Lightwave Technol., Vol. 34, no. 8, p. 1516 (2016).

7. ITU-T recommendation G.975.1 (2004). 